\title{
Improved Charge Pump with Reduced Reverse Current
}

\author{
Kiuk Gwak, Sang-Gug Lee, and Seung-Tak Ryu
}

\begin{abstract}
A highly efficient charge pump that minimizes the reverse charge sharing current (in short, reverse current) is proposed. The charge pump employs auxiliary capacitors and diode-connected MOSFET along with an early clock to drive the charge transfer switches; this new method provides better isolation between stages. As a result, the amount of reverse current is reduced greatly and the clock driver can be designed with reduced transition slope. As a proof of the concept, a $1.1 \mathrm{~V}$-to-9.8 V charge pump was designed in a $0.35 \mu \mathrm{m} 18 \mathrm{~V}$ CMOS technology. The proposed architecture shows $1.6 \mathrm{~V} \sim$ $3.5 \mathrm{~V}$ higher output voltage compared with the previously reported architecture.
\end{abstract}

Index Terms - Charge pump, Reverse current, DCDC converter, MEMS microphone

\section{INTRODUCTION}

Charge pump is one type of DC-DC converter that is often used to produce higher DC voltage from a given supply. The simple structure of it makes charge pump popular in applications such as read/write operation of EEPROM and MEMS MIC, which have relatively low load current with moderate ripple requirements [1-8]. Especially for the applications that require extremely low load current such as for capacitive load (e.g. MEMS microphone which consumes only few nA), charge pump is an ideal candidate for supply generation because of its compactness and low power consumption.

Among various designs, Wu and Chang's architecture

Manuscript received Jan. 5, 2012; revised Mar. 23, 2012. Department of Electrical Engineering, KAIST, Daejeon, Korea E-mail : stryu@ee.kaist.ac.kr
[2], based on Dickson's charge pump [1], is a representative one. They developed a charge pump that replaces diodes with switches to achieve higher voltage conversion ratio. However, the reverse current in that structure, caused by the finite rising time of the clock, limits the voltage conversion gain and makes it difficult to predict the output voltage. Thus, for higher output voltage, more stages than the theoretically expected is required.

Several advanced designs with focuses on CMOS reliability, higher load current driving ability, and small chip area [3-5] have been published. Nevertheless, those designs are not suitable for the applications where low input voltage is given and when transistors have relatively high threshold voltages. For example, in Ker's design [5], the maximum gate-source voltage was restricted under VDD. Hence, unless VDD is over the threshold voltage of a given transistor, the charge pump is not able to perform properly. Further modifications [6, 7] show the higher power efficiency and smaller chip area, but they require a complicated timing control of the clock signal.

In this work, we suggest an improved structure of [2] by eliminating the reverse current path with a modified switch control circuit.

\section{Previous Charge Pump}

Three consecutive stages of the charge pump of [2] are shown in Fig. 1. Assume the circuit is in steady-state. When CLK changes from 0 to $\mathrm{V}_{\mathrm{DD}}$ (when CLKB, the inverse of CLK, falls), the $n^{\text {th }}$ stage output, $V_{n}$, increases by $\Delta \mathrm{V}$ and the voltages of neighboring stages reduce by the same amount. Consequently, the auxiliary NMOS switch, $\mathrm{SW}_{\mathrm{auxN}}$, for the control of $\mathrm{n}^{\text {th }}$ charge transfer 


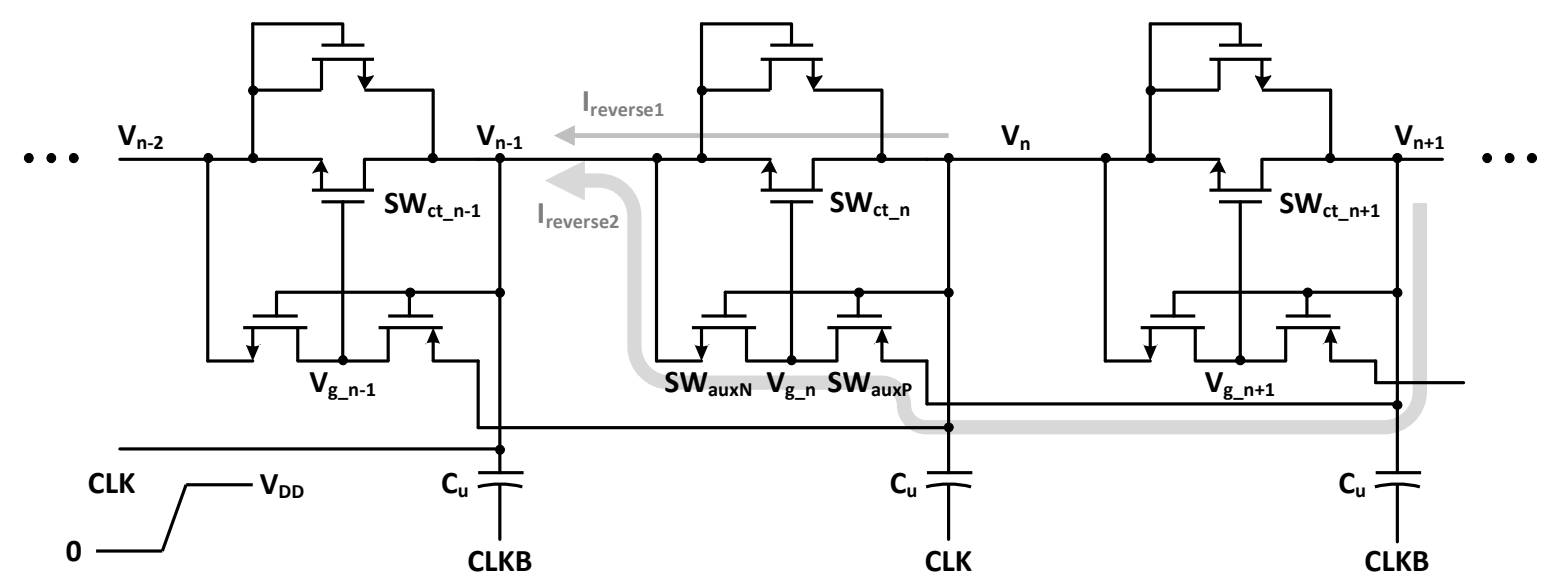

Fig. 1. Circuit diagram of Wu and Chang's charge pump [2].

switch, $\mathrm{SW}_{\text {ct_n }}$, turns on by $\mathrm{V}_{\mathrm{n}}-\mathrm{V}_{\mathrm{n}-1}=2 \Delta \mathrm{V}$ and the PMOS switch, $\mathrm{SW}_{\text {auxp }}$, turns off. Thus, $\mathrm{V}_{\mathrm{g} \_\mathrm{n}}$ falls from $\mathrm{V}_{\mathrm{n}+1}$ to $\mathrm{V}_{\mathrm{n}-1}$ and turns off $\mathrm{SW}_{\mathrm{ct} \_\mathrm{n}}$, and $\mathrm{V}_{\mathrm{g} \_\mathrm{n}-1}$ and $\mathrm{V}_{\mathrm{g} \_\mathrm{n}+1}$ rise to turn on the $(n-1)^{\text {th }}$ and $(n+1)^{\text {th }}$ charge transfer switches, $\mathrm{SW}_{\text {ct } \_-1}$ and $\mathrm{SW}_{\text {ct } \_n+1}$. Hence, the $(\mathrm{n}+1)^{\text {th }}$ stage is charged up to the peak voltage of $V_{n}$. Similarly, when CLK falls (CLKB rises), $\mathrm{V}_{\mathrm{n}+1}$ is charged by $\Delta \mathrm{V}$. Thus, the output voltage of the charge pump with $\mathrm{n}$ stages is determined as

$$
\mathrm{V}_{\text {out }}=\mathrm{V}_{\mathrm{DD}}-\mathrm{V}_{\mathrm{d}}+\mathrm{n} \Delta \mathrm{V}
$$

where $V_{d}$ is the voltage drop of diode, which is required at the output stage [2]. Ideally, $\Delta \mathrm{V}$ should be close to $\mathrm{V}_{\mathrm{DD}}$. However, the parasitic capacitance at each node and the unwanted reverse currents cause $\Delta \mathrm{V}$ to be smaller than $V_{D D}$. Since the parasitic capacitance effect is not significant and can be overcome with ease by increasing unit capacitance $\mathrm{C}_{\mathrm{u}}$, the reverse current effect becomes the dominant loss factor.

Fig. 2 shows detailed voltage waveform of each node of the circuit in Fig. 1 when CLK changes from 0 to $V_{D D}$. At $\mathrm{t}=\mathrm{t}_{0}, \mathrm{~V}_{\mathrm{n}}$ and $\mathrm{V}_{\mathrm{n}-1}$ have the same value due to the closed $\mathrm{SW}_{\text {ct_n }}$ with $\mathrm{V}_{\mathrm{g} \_\mathrm{n}}=\mathrm{V}_{\mathrm{n}+1}$, and $\mathrm{V}_{\mathrm{n}+1}$ is higher than $\mathrm{V}_{\mathrm{n}}$ by $2 \Delta \mathrm{V}$. As CLK rises (CLKB falls), $\mathrm{V}_{\mathrm{n}}$ increases, and $V_{n+1}$ and $V_{n-1}$ deviate from each other via the finite resistance of $\mathrm{SW}_{\mathrm{ct} \_n}$. Ideally, $\mathrm{SW}_{\text {ct_n }}$ must be turned off completely as soon as CLK changes to high in order to prevent the reverse current from $V_{n}$ to $V_{n-1}$, which reduces the charge pumping efficiency. This is the reverse current, $\mathrm{I}_{\text {reverse }}$ in Fig. 1. However, $\mathrm{SW}_{\text {auxN }}$ does not turn on until $\mathrm{V}_{\mathrm{n}}-\mathrm{V}_{\mathrm{n}-1}\left(=\mathrm{V}_{\mathrm{GS}}\right.$ of $\left.\mathrm{SW}_{\mathrm{auxN}}\right)$ reaches the

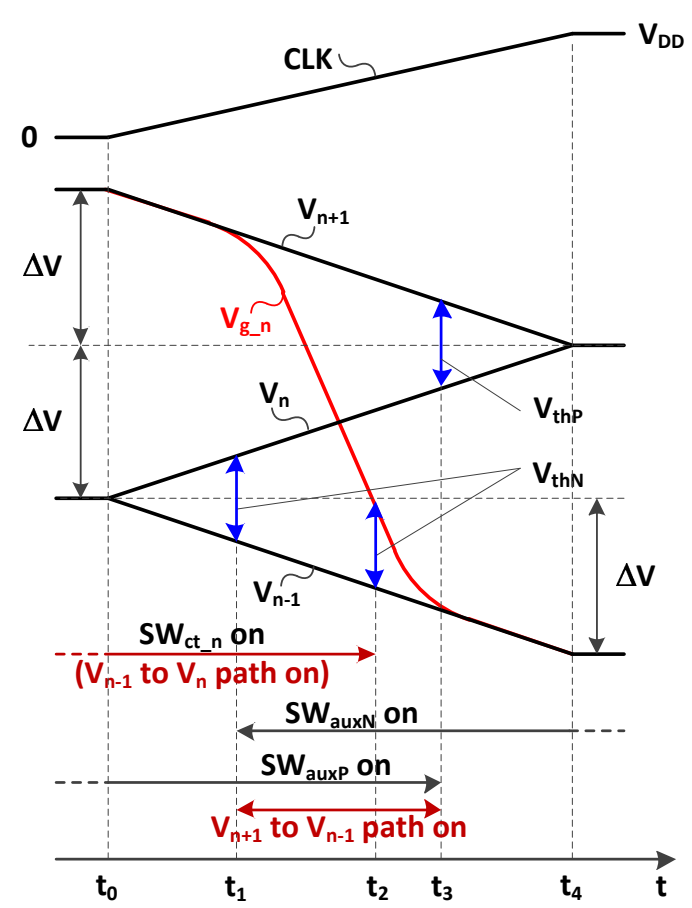

Fig. 2. Operation of the charge pump in Fig. 1.

NMOS threshold, $V_{\text {thN }}$, and, thus, $V_{\mathrm{g}_{\_} \mathrm{n}}$ is tied to $\mathrm{V}_{\mathrm{n}+1}$ and, thus, $\mathrm{SW}_{\mathrm{ct} \_\mathrm{n}}$ stays on. As time passes $\mathrm{t}=\mathrm{t}_{1}, \mathrm{~V}_{\mathrm{n}}-\mathrm{V}_{\mathrm{n}-1}$ becomes larger than $\mathrm{V}_{\mathrm{thN}}$ and $\mathrm{SW}_{\mathrm{auxN}}$ turns on. Since $\mathrm{SW}_{\text {auxP }}$ is still on strongly, this makes another reverse current, $\mathrm{I}_{\text {reverse2 }}$, from $\mathrm{V}_{\mathrm{n}+1}$ to $\mathrm{V}_{\mathrm{n}-1}$ via $\mathrm{SW}_{\text {auxp }}$ and $\mathrm{SW}_{\text {auxN }}$ while $I_{\text {reversel }}$ still exists. After $t=t_{2}, S_{\text {ct_n }}$ turns off $\left(V_{\mathrm{g} \mathrm{n}}-\mathrm{V}_{\mathrm{n}-1}<\mathrm{V}_{\text {thN }}\right)$ and $\mathrm{I}_{\text {reversel }}$ becomes zero. When $\mathrm{t}>\mathrm{t}_{3}$, $\mathrm{SW}_{\text {auxp }}$ turns off $\left(\mathrm{V}_{\mathrm{n}+1}-\mathrm{V}_{\mathrm{n}}<\mathrm{V}_{\text {thP }}\right)$ and $\mathrm{I}_{\text {reverse2 }}$ becomes zero. Here, $V_{\text {thP }}$ is the threshold voltage of PMOS. To sum up, the charge pumping stages work ideally only after $\mathrm{t}=\mathrm{t}_{3}$ with no reverse current. 


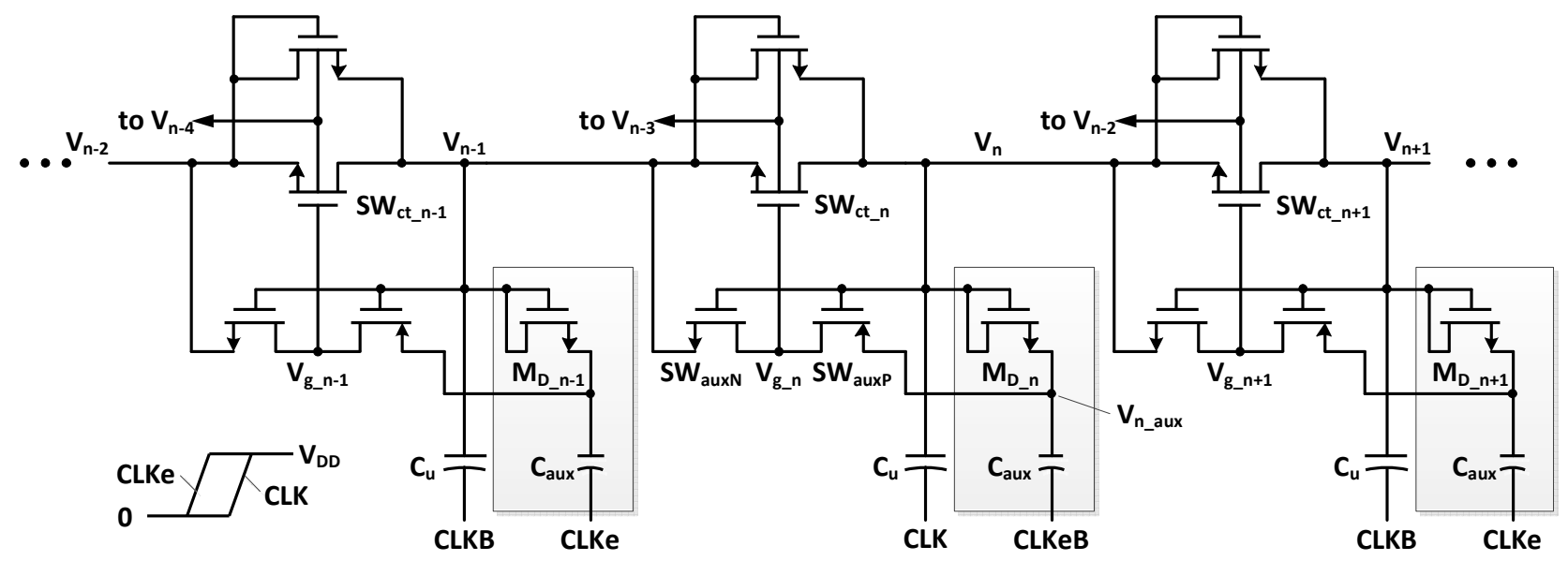

Fig. 3. Circuit diagram of the proposed charge pump.

Note that $\mathrm{I}_{\text {reverse2 }}$ is the most serious problem in $\mathrm{Wu}$ and Chang's structure because it makes a short circuit current through the stages driven by $\operatorname{CLKB}\left(\ldots, \mathrm{V}_{\mathrm{n}-3}, \mathrm{~V}_{\mathrm{n}-1}\right.$, $\left.\mathrm{V}_{\mathrm{n}+1}, \mathrm{~V}_{\mathrm{n}+3}, \ldots\right)$ and degrades the charge pumping gain seriously. Likewise, stages driven by CLK $\left(\ldots, V_{n-2}, V_{n}\right.$, $\left.\mathrm{V}_{\mathrm{n}+2}, \ldots\right)$ are found to short each other during the clock transition periods. Hence, to reduce the amount of the reverse current, the rising time, $t_{r}$, of the clock should be minimized by employing large clock buffers, which increases the dynamic power consumption and circuit noise.

\section{Proposed Charge Pump}

In this work, in order to reduce the reverse currents in $\mathrm{Wu}$ and Chang's structure, a modified architecture is proposed as shown in Fig. 3. In order to break the long short-circuit current $\left(\mathrm{I}_{\text {reverse } 2}\right)$ path driven by CLK and CLKB, control signals from the next stages, the proposed circuit employs an additional clock signal, CLKe, which is an early-time version of CLK. In addition, in the circuit, a diode-connected transistor, $\mathrm{M}_{\mathrm{D} \mathrm{k}}$, and an auxiliary capacitor, $\mathrm{C}_{\mathrm{aux}}$, are added in each stage. Note that $\mathrm{C}_{\mathrm{aux}}$ can be designed to be much smaller than $\mathrm{C}_{\mathrm{u}}$ because the parasitic capacitance at every node driven by $\mathrm{C}_{\mathrm{aux}}$ is small and $\mathrm{C}_{\mathrm{aux}}$ does not provide current to the load.

The operation of the proposed architecture is illustrated in Fig. 4. In steady-state, $V_{n \_a u x}$ (refere to Fig. 3) has the same value as $V_{n+1}-V_{t h N}$ because of the diodeconnected NMOS, $\mathrm{M}_{\mathrm{D}_{\mathrm{n}} \mathrm{n}}$ and the clock configurations.

For $\mathrm{t}<\mathrm{t}_{0}, \mathrm{CLK}=0$ and the inverse of the early clock,

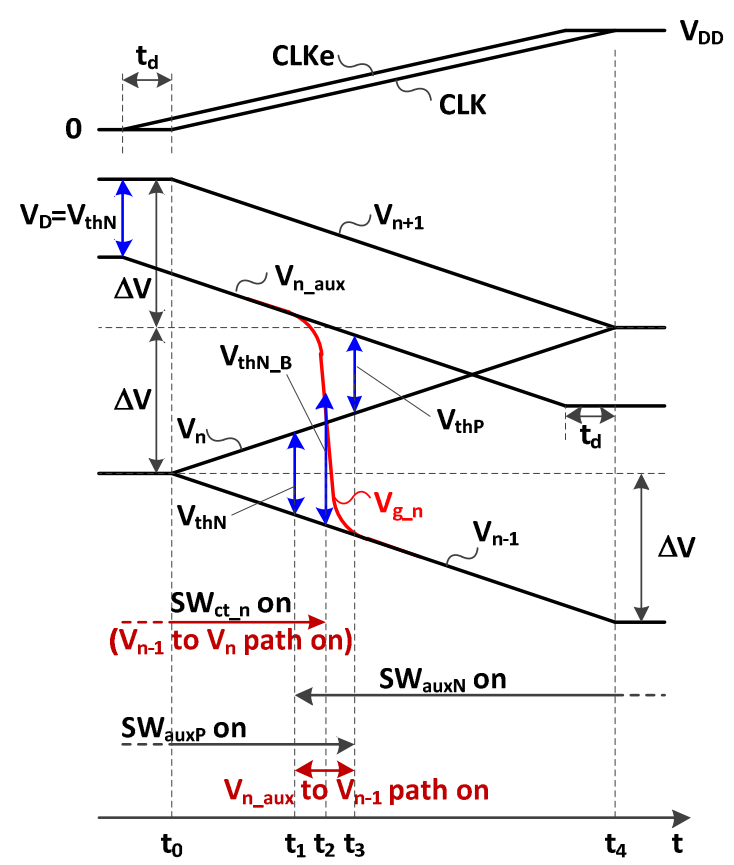

Fig. 4. Operation of the charge pump in Fig. 3.

CLKeB, starts to fall and, thus, $\mathrm{V}_{\mathrm{g} \_\mathrm{n}}$ decreases. After a delay of $t_{d}\left(\right.$ at $\left.t=t_{0}\right)$ CLK starts to rise and, thus, $V_{n}$ and $\mathrm{V}_{\mathrm{n}-1}$ begins to increase and decrease, respectively. As long as $\left(\mathrm{V}_{\mathrm{g}_{-} \mathrm{n}}-\mathrm{V}_{\mathrm{n}-1}\right)$ is higher than the threshold of $\mathrm{SW}_{\text {ct_ } \mathrm{n}}$ $\left(\mathrm{V}_{\text {thN_B }}\right), \mathrm{SW}_{\text {ct_n }}$ stays on and the reverse current flows from $V_{n}$ to $V_{n-1}\left(I_{\text {reversel }}\right.$ as in Fig. 1). However, unlike the previous work [2] in this work, intentionally generated body effect reduces the reverse current through $\mathrm{SW}_{\text {ct_n }}$ by increasing its threshold voltage from $\mathrm{V}_{\text {thN }}$ to $\mathrm{V}_{\text {thN_B }}$. Note that this design uses a deep n-well process and the body terminal of $\mathrm{SW}_{\mathrm{ct} \mathrm{n}}$ is connected to $\mathrm{V}_{\mathrm{n} 3}$ for the increased 
threshold and to guarantee a constant body-to-source voltage of $2 \Delta \mathrm{V}$. This choice has been made so that the on-resistance of $\mathrm{SW}_{\mathrm{ct} \_n}$ increases to reduce the reverse current while guaranteeing that $\mathrm{V}_{\text {thN_B }}$ is smaller than $2 \Delta \mathrm{V}$. This approach also solves the increasing threshold problem of $\mathrm{SW}_{\text {ct_n }}$ as stage goes to the output in [2].

When $\mathrm{t}=\mathrm{t}_{1}, \mathrm{SW}_{\text {auxN }}$ starts to turn on, similarly to the case in Figs. 1 and 2, because its $\mathrm{V}_{\mathrm{GS}}$ exceeds the threshold, $\mathrm{V}_{\mathrm{thN}}$. However, since stages are not connected to each other via the auxiliary switches, the reverse current through them ( $I_{\text {revers2 }}$ in Fig. 1) is completely eliminated. Even though some amount of charge can be shared between $V_{n \_a u x}$ and $V_{n-1}$, the effect is negligible because $\mathrm{V}_{\mathrm{n} \_ \text {aux }}$ always has higher voltage than $\mathrm{V}_{\mathrm{n}-1}$ and their capacitance difference is huge $\left(\mathrm{C}_{\mathrm{u}}>\mathrm{C}_{\mathrm{aux}}\right)$. Actually, this helps turning off $\mathrm{SW}_{\mathrm{ct} \_n}$ by dropping $\mathrm{V}_{\mathrm{g}_{\_} \mathrm{n}}$ earlier than the ideal case. Furthermore, if $t_{d}$ becomes sufficiently long, $\mathrm{SW}_{\text {auxP }}$ turns off earlier than $\mathrm{SW}_{\text {auxN }}$ 's turn-on, which removes the charge sharing between $\mathrm{C}_{\mathrm{u}}$ and $\mathrm{C}_{\text {aux }}$.

When $t$ passes $t_{2}, V_{g_{n} n}-V_{n-1}$ becomes smaller than $\mathrm{V}_{\text {thN_B }}$ and $\mathrm{SW}_{\text {ct_n }}$ turns off. Note that the time for $\mathrm{I}_{\text {reverse1 }}$ is shorter than that in [2] not only because the threshold voltage of $\mathrm{SW}_{\text {ct_n }}$ is increased from $\mathrm{V}_{\text {thN }}$ to $\mathrm{V}_{\text {thN_B }}$ but also because the starting level of $V_{g_{\_} n}$ (i.e. $V_{n_{\_} \text {aux }}$ when CLKeB goes low) is lowered by the diode voltage drop $\mathrm{V}_{\mathrm{D}}=\mathrm{V}_{\text {thN }}$ and CLKeB starts to decrease earlier. As $\mathrm{t}$ passes $\mathrm{t}_{3}, \mathrm{SW}_{\text {auxp }}$ turns off due to $\mathrm{V}_{\mathrm{g}_{-} \mathrm{n}}-\mathrm{V}_{\mathrm{n}-1}<\mathrm{V}_{\text {thN_B }}$. For the remaining time until CLK reaches $\mathrm{V}_{\mathrm{DD}}$, the charge pump works ideally.

Consequently, compared to $\mathrm{Wu}$ and Chang's architecture the reverse currents through all the paths are reduced significantly in the proposed work.

\section{RESULTS}

The proposed charge pump was designed for a 0.35 $\mu \mathrm{m}$ 1P4M $18 \mathrm{~V}$ CMOS technology. Typical threshold voltage of NMOS is $0.8 \mathrm{~V}$ and that of PMOS is $1.1 \mathrm{~V}$. For all designs, 18V PIP capacitors are used. For both charge pumps, [2] and the proposed one, the total capacitance was kept the same for fair comparison.

To verify the effect of the reduced reverse currents, rising time of CLK, $\mathrm{t}_{\mathrm{r}}$, was varied. At zero load current (assumed capacitive load driving such as MEMS microphone), the output voltages after the last stage diode are plotted in Figs. 5 and 6 under a $1.1 \mathrm{~V}$ input
$\left(\mathrm{V}_{\mathrm{DD}}\right)$ condition and $5 \mathrm{MHz}$ clock frequency.

Fig. 5 shows the simulated outputs of 10-stage charge pumps with the structure in [2] and the proposed one. Solid and dotted lines represent the proposed and $\mathrm{Wu}$ and Chang's architecture, respectively. As the unit capacitance increases, the effect of the parasitic capacitance at each node is reduced and thus the output voltage increases.

For the proposed architecture, the output voltage variation is less than $1 \%$ when the rising/falling time of the clock varies from $0 \mathrm{~s}$ to $4 \mathrm{~ns}$. On the other hand, for $\mathrm{Wu}$ and Chang's architecture, the output voltage varies over $30 \%$ of the expected value due to the reverse current caused by the finite clock rising time of the clock. The unit capacitance $\left(\mathrm{C}_{\mathrm{u}}\right)$ and auxiliary capacitance $\left(\mathrm{C}_{\text {aux }}\right)$ of the proposed architecture is chosen to be $294 \mathrm{fF}$ and 48 $\mathrm{fF}$, respectively. Consequently, the unit capacitance of $344 \mathrm{fF}$ is used for the architecture of [2].

Fig. 6 compares the output voltages of the two designs for different number of stages. Apparently, $\Delta \mathrm{V}$ of the proposed architecture stays near $\mathrm{V}_{\mathrm{DD}}$ in all conditions. On the other hand, $\Delta V$ of [2] is quite smaller than $V_{D D}$ and seriously decreases as $t_{r}$ increases. Note that $t_{d}$ is set to be half of $t_{r}$ in this simulation. As long as $t_{d}$ is over half of $t_{r}$, the accuracy of $t_{d}$ barely affects the performance of the charge pump.

Layout of the proposed and $\mathrm{Wu}$ and Chang's are shown in Fig. 7. Although the unit capacitance of the proposed architecture(top) is smaller, total area including auxiliary capacitors is similar for both the proposed and Wu and Chang's architecture (bottom), $182 \times 125 \mu^{2}$.

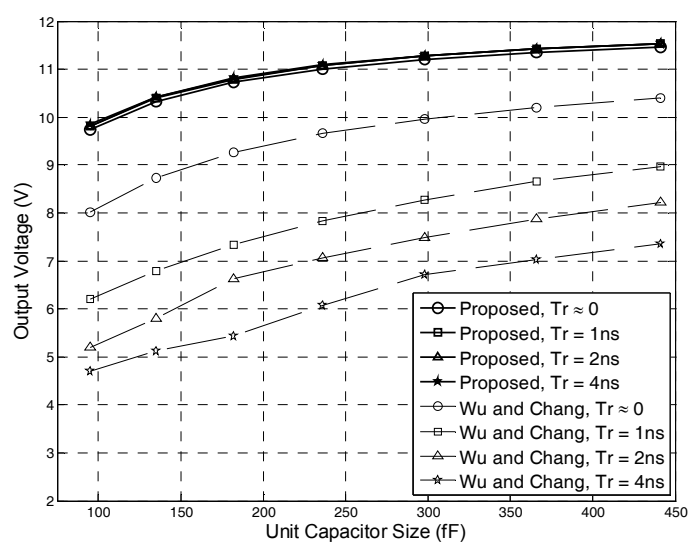

Fig. 5. Simulated output voltages of 10 -stage of $\mathrm{Wu}$ and Chang's charge pump and the proposed charge pump with varying unit capacitance and $1.1 \mathrm{~V} \mathrm{~V}_{\mathrm{DD}}$ and $1 \mathrm{pF}$ capacitive load. 


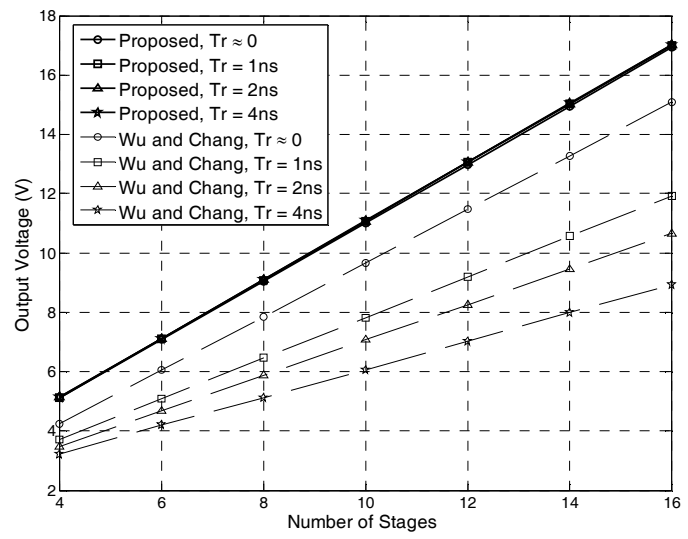

Fig. 6. Simulation results for $\mathrm{Wu}$ and Chang's and the proposed charge pump with various $t_{r}$ and the number of stages when $1 \mathrm{pF}$ capacitive load is used.

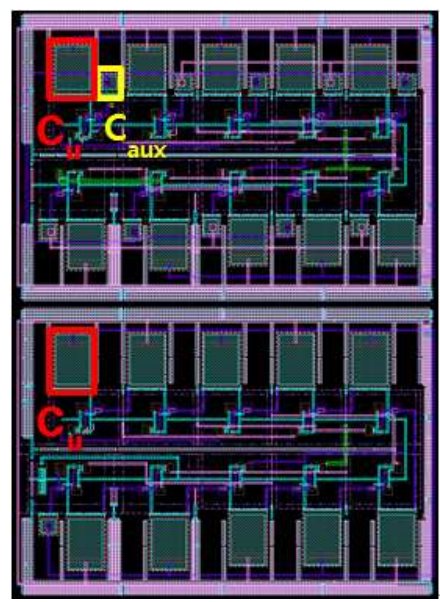

Fig. 7. Layout of the proposed charge pump (top) and $\mathrm{Wu}$ and Chang's charge pump (bottom). The sizes of outlines for both layout are about $182 \times 125 \mu^{2}$.

Fig. 8 shows the post-simulation results of both charge pumps with 10 stages with $5 \mathrm{MHz}$ clock. When the load current is zero, compared to the pre-simulation results in Fig. 5, the output voltage is degraded approximately by $1.3 \mathrm{~V}$ due to the parasitic capacitance of the body connections of NMOS and PMOS. Because small unit capacitance is employed, the output voltage degrades rapidly as the load current increases. However, the proposed architecture shows higher output voltages than [2] in all cases. Note that, as shown in Fig. 5, 6, 7, and 8, the performance of the proposed architecture is not limited by the small unit capacitance and no load condition.

Consequently, it is verified that the proposed architecture outperforms the conventional charge pump

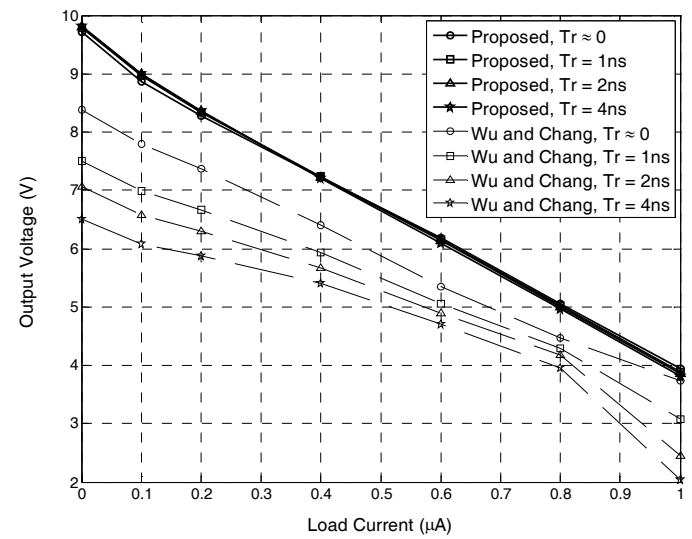

Fig. 8. Post-simulation results for 10 stages of $\mathrm{Wu}$ and Chang's charge pump and the proposed charge pump with various $t_{\mathrm{r}}$ and load current with $1 \mathrm{pF}$ capacitor in parallel. 5 $\mathrm{MHz}$ clock frequency is used, and $\mathrm{t}_{\mathrm{d}}$ is set to the half of $\mathrm{t}_{\mathrm{r}}$.

owing to the reduced reverse current. This advantage allows the designer to use a smaller clock buffer and less number of stages for lower power consumption and small area. Since the proposed architecture is insensitive to the slope of the clock edge, the discrepancy between the design and measurement results is decreased. Unfortunately, however, we could not measure the fabricated chip because of the error in I/O design.

\section{Conclusions}

A new charge pump architecture for reduced reverse current is presented. Based on $\mathrm{Wu}$ and Chang's architecture, the proposed architecture employs an additional diode and capacitor to isolate each stage. In addition, intentional body bias and an early clock are employed to further reduce the reverse current. As a result, the proposed charge pump has higher output voltage and robustness to the variation of the clock with a chance of lower power design.

\section{ACKNOWLEDGMENTS}

This work was supported by the National Research Foundation of Korea Grant funded by the Korea government (MEST) (No. NRF-2010-0001546). The CAD tools were supported by IDEC of KAIST. 


\section{REFERENCES}

[1] John F. Dickson, "On-Chip High-Voltage Generation in MNOS Integrated Circuits Using an Improved Voltage Multiplier Technique," SolidState Circuits, IEEE Journal of, Vol.11, No.3, pp. 374-378, Jun., 1976.

[2] Jieh-Tsorng $\mathrm{Wu}$ and Kuen-Long Chang, "MOS Charge Pumps for Low-Voltage Operation," SolidState Circuits, IEEE Journal of, Vol.33, No.3, pp.592-597, Apr., 1998.

[3] Roberto Pelliconi, et al., "Power Efficient Charge Pump in Deep Submicron Standard CMOS Technology," Solid-State Circuits, IEEE Journal of, Vol.38, No.6, pp.1068-1071, Jun., 2003.

[4] Ming-Dou ker, Shih-Lun Chen, and Chia-Shen Tsai, "Design of Charge Pump Circuit with Consideration of Gate-Oxide Reliability in LowVoltage CMOS Processes," Solid-State Circuits, IEEE Journal of, Vol.41, No.5, pp.1100-1107, May, 2006.

[5] Kyeong-Pil Kang and Kyeong-Sik Min, "Charge Pump Circuits with Low Area and High Power Efficiency for Memory Applications," Semiconductor Technology and Science, Journal of, Vol.6, No.4, pp.257-263, Dec., 2006.

[6] Takanori Yamazoe, Hisanobu Ishida, and Yasutaka Nihongi, "A Charge Pump that Generates Positive and Negative High Voltages with Low PowerSupply Voltage and Low Power Consumption for Non-volatile Memories," Circuits and Systems, IEEE International Symposium on, pp.988-991, 2009.

[7] Yi-Hsin Weng, Hui-Wen Tsai, and Ming-Dou Ker, "Design of Charge Pump Circuit in Low-Voltage CMOS Process with Suppressed Return-Back Leakage Current," IC Design and Technology, IEEE International Conference on, pp.155-158, 2010.

[8] Du-Hwi Kim, Ji-Hye Jang, Liyan Jin, Pan-Bong Ha, and Young-Hee Kim, "Design of an EEPROM for a MCU with the Wide Voltage Range," Semiconductor Technology and Science, Journal of, Vol.10, No.4, pp.316-324, Dec., 2010.

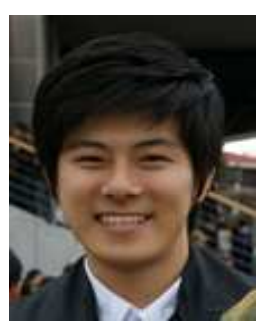

Kiuk Gwak received the B.S. and M.S. degree in electrical engineering from Korea Advanced Institute of Science and Technology (KAIST), Korea, in 2010 and 2011, respectively. He is currently pursuing the Ph.D. degree at the department of electrical and electrical engineering in KAIST. His interest includes analog circuit and data converter design and brain reverse engineering.

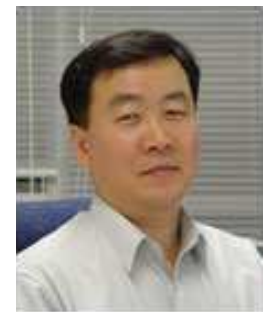

Sang-Gug Lee received a B.S. degree in electronic engineering from Kyungpook National University, Korea, in 1981, and M.S. and Ph.D. degrees in electrical engineering at the University of Florida, Gainesville, in 1989 and 1992, respectively. In 1992, he joined Harris Semiconductor, Melbourne, Florida, where he was engaged in silicon-based RFIC design. From 1995 to 1998, he was with Handong University, Pohang, Korea, as an Assistant Professor in the School of Computer and Electrical Engineering. From 1998 to 2009, he was with the Information and Communications University, Daejeon, Korea, where he became a Professor. Since 2009, he has been with the Korea Advanced Institute of Science and Technology (KAIST), Daejeon, Korea, in the Department of Electrical Engineering as a Professor. His research interests include CMOS-based RF, analog, and mixed mode IC design for various radio transceiver applications. More recently, his research has focused on low-power transceivers and extreme high-frequency $(\mathrm{THz})$ circuit design based on CMOS technology, and display and energy-harvesting IC design. 


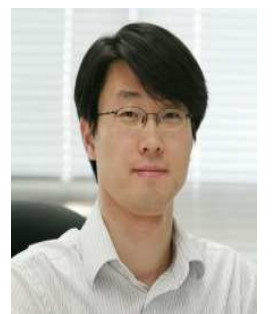

Seung-Tak Ryu (M'06) received the

B.S. degree in electrical engineering from Kyungpook National University, Korea, in 1997, and the M.S. and Ph.D. degrees from Korea Advanced Institute of Science and Technology (KAIST) in 1999 and 2004, respectively. From 2001 to 2002, he was with University of California at San Diego as a visiting researcher sponsored through the Brain Korea 21 (BK21) program. In 2004, he joined Samsung Electronics, Kiheung, Korea where he was involved in mixed-signal IP design. From 2007 to 2009, he was with the Information and Communications University (ICU), Daejeon, Korea, as an Assistant Professor. Since 2009, he has been with Korea Advanced Institute of Science and Technology (KAIST), Daejeon, Korea, in the Department of Electrical Engineering as an Assistant Professor. His research interests include analog and mixed signal IC design with an emphasis on data converters. 